\title{
SCREENING COMMERCIAL DRIVERS FOR OBSTRUCTIVE SLEEP APNEA: VALIDATION OF STOP-BANG QUESTIONNAIRE
}

\section{MARTIN B. POPEVIĆ ${ }^{1,2}$, ANDJELA MILOVANOVIĆ ${ }^{1,3}$, LJUDMILA NAGORNI-OBRADOVIĆ ${ }^{1,4}$, DEJAN NEŠIĆ ${ }^{1,5}$, JOVICA MILOVANOVIĆ ${ }^{1,6}$, and ALEKSANDAR P.S. MILOVANOVIĆ ${ }^{1,2}$}

${ }^{1}$ University of Belgrade, Belgrade, Serbia

Faculty of Medicine

${ }^{2}$ Serbian Institute of Occupational Health "Dr Dragomir Karajović," Belgrade, Serbia

${ }^{3}$ Clinical Centre of Serbia, Belgrade, Serbia

Clinic for Physical Medicine and Rehabilitation

${ }^{4}$ Clinical Centre of Serbia, Belgrade, Serbia

Clinic for Pulmonology

${ }^{5}$ Institute of Medical Physiology, Belgrade, Serbia

${ }^{6}$ Clinical Centre of Serbia, Belgrade, Serbia

Clinic for Otorhinolaryngology and Maxillofacial Surgery

\begin{abstract}
Objectives: The main aim has been to examine psychometric properties of STOP-Bang (snoring, tiredness, observed apnea, high blood pressure, body mass index (BMI), age, neck circumference, male gender) scoring model (Serbian translation), an obstructive sleep apnea (OSA) screening tool, in a sample of commercial drivers. Material and Methods: After formal translation, validation was performed on a sample of bus and truck drivers evaluating test-retest reliability, construct and criterion validity. Overnight polysomnography or cardiorespiratory polygraphy were used for OSA diagnosis purposes. Results: One hundred male participants, 24-62 years old, were included. STOP-Bang classified 69\% as potential OSA patients. Polysomnography identified OSA in $57 \%$ of the sample. Test-retest reliability (Cohen's $x=0.89$ ) was adequate. STOP-Bang score was significantly correlated to apnea-hypopnea index (AHI) and OSA severity. Sensitivity was $100 \%$ for $\mathrm{AHI} \geq 15$, highest specificity was $53.5 \%$ (AHI $\geq 5$ ). Conclusions: STOP-Bang showed good measurement properties, supporting its further use in OSA screening of commercial drivers. Int J Occup Med Environ Health 2016;30(5):751-761
\end{abstract}

Key words:

Sleep apnea, Questionnaires, Polysomnography, Obstructive, Validation studies, Commercial drivers

Funding: project of the Ministry of Education and Science of Serbia ON 175081 (2011-2014) "Significance of early diagnosis of obstructive sleep apnoea syndrome in professional drivers operating motor vehicles." Project manager: Prof. Aleksandar Milovanović.

Received: January 26, 2016. Accepted: August 11, 2016.

Corresponding author: M.B. Popević, Serbian Institute of Occupational Health "Dr Dragomir Karajović," Deligradska 29, 11000 Belgrade, Serbia (e-mail: popevic.martin@gmail.com). 


\section{INTRODUCTION}

Obstructive sleep apnea (OSA) is a common chronic disorder defined by characteristic signs and symptoms (daytime sleepiness, loud snoring, witnessed breathing pauses or awakenings) in the presence of at least 5 obstructive respiratory events per hour of sleep [1].

Estimated prevalence of moderate to severe OSA in the adult population is $13 \%$ in men and $6 \%$ in women [2], but it is believed to be significantly higher (25-50\%) in the population of commercial drivers [3-5]. If not diagnosed or treated, OSA may cause or aggravate cardiovascular, metabolic and psychiatric disorders [6-9], as well as all cause mortality and mortality from coronary artery disease $[10,11]$. There is strong evidence linking OSA and permanent impairment of cognitive functions, including attention, executive functions and psychomotor speed and coordination [12].

Drivers of motor vehicles, especially commercial drivers are considered to be a population in high risk of OSA, with a 2- to 5-fold greater risk of traffic accidents and a 3-5 time stronger chance of personal injury $[1,3,4,13,14]$. The gravity of this problem has been recently recognized by regulatory authorities in the European Union (EU), so a new directive 2014/85/EU [15] regulating OSA and fitness to drive for amateur and commercial drivers has been adopted. Major implication of this directive is that all drivers must be screened for OSA risk, drivers in high risk of moderate and severe OSA must be examined, and drivers with diagnosed OSA must receive regular treatment and follow-up [15].

The gold standard for OSA diagnosis is an overnight attended complete polysomnography (type I PSG), but the procedure is time-consuming and expensive, and capacities of diagnostic facilities are limited. There is a strong demand for a good OSA screening tool that may help physicians determine who among their patients needs to be sent to a sleep clinic first. Use of self-reported questionnaires is a preferred initial step in clinical and research settings $[16,17]$.
Epworth sleepiness scale is used for detecting levels of possible sleepiness (scale: $0-3$ ) in 8 different situations. Epworth sleepiness scale (ESS) score $\geq 10$ is considered significant [18]. It is widely used, but the real value for patients with OSA is still not clear, since many of them do not have or do not recognize excessive sleepiness [19]. STOP-Bang (snoring, tiredness, observed apnea, high blood pressure, body mass index (BMI), age, neck circumference, and male gender) questionnaire is an 8-item check list with yes/no answers created to screen surgical patients for risk of OSA. Patients with 3 or more positive answers are considered high risk [20]. STOP-Bang has been primarily used for prediction of OSA-related perioperative risks, but also for OSA screening in community settings [21], for patients with progressive chronic diseases such as multiple sclerosis [22] or kidney failure [23], for army veterans [24], and patients referred to sleep clinics [25].

Usefulness of questionnaires is of special interest in occupational health screening of high risk groups such as commercial drivers. Several studies have confirmed that commercial drivers underreport sleep disorder symptoms, like excessive daytime sleepiness [26], in order to avoid potential economic and occupational consequences [27]. STOP-Bang has been used [28-30] and even recommended [31] for screening professional drivers, but the psychometric properties in this high risk population have rarely been reported [28].

The main aim of our study has been to validate a STOPBang scoring model (formal Serbian translation) in a sample of commercial drivers in order to test its usefulness in occupational health screening for OSA.

\section{MATERIAL AND METHODS}

\section{Translation study}

After obtaining permission from the author, we performed the translation and cross cultural adaptation of STOPBang questionnaire to Serbian language according to guidelines by Beaton et al. [32]. 
Two forward translations were produced by accredited medical English translators, not involved in the study. Backward translation was performed by a professional bilingual translator, native in the English language, blinded to the original questionnaire. Backward translation was then compared to the original version, and discussed by an expert panel of clinicians. Pre-final version was tested for equivalence of terms with the original on a sample of 20 bilingual subjects (healthcare workers, undergraduate medical students), and after that, on a sample of $10 \mathrm{pa}-$ tients with untreated obstructive sleep apnea.

Following a cognitive debriefing, a final Serbian version of the STOP-Bang scoring model was created. A detailed report on the linguistic validation procedure was sent to the author of the original questionnaire.

\section{Validation study}

\section{Population}

One hundred commercial drivers, all male, in the age range of 24-62 years old, working in several public transportation companies in Belgrade, Serbia were included. Subjects were a part of a larger study examining sleepiness, presence of OSA and traffic accidents in professional drivers, which included 396 out of 500 employed drivers (response rate 79.2\%). First 100 drivers that underwent polysomnography (by random choice) were included in the validation study. Informed consents were obtained from all participants. Inclusion criteria were that subjects had to be employed as commercial drivers in transportation of passengers/goods, previously not diagnosed with a sleep disorder of any kind, clearly understood the study protocol and agreed to participate.

\section{Study design}

In the first phase, all subjects were approached at bus or truck depots where they retrieved their vehicles. After detailed explanation, all subjects completed a generic questionnaire with questions about their sleep, work and life habits, personal and family health history, as well as the Serbian version of STOP-Bang questionnaire and Epworth sleepiness scale. In the second phase, subjects were admitted to the Serbian Institute of Occupational Health, retested with the same set of questionnaires and examined by a physician experienced in sleep disorders. Eighty-nine patients underwent a full night attended complete polysomnography (PSG) (Type I PSG). Out of 11 patients who performed full night attended cardiorespiratory polysomnography (modified Type III PSG), a negative diagnostic result was seen in 2 patients. Since these studies were performed in attended settings, they were accepted as diagnostic, with no need for Type I PSG.

\section{Instruments}

\section{STOP-Bang questionnaire (SBQ)}

The STOP-Bang scoring model consists of 2 parts. The subjective part is STOP questionnaire with 4 yes/no questions on perceived presence of loud snoring, excessive daytime tiredness, observed apnea, and treated/untreated high blood pressure. One point is assigned for each positive answer. The objective part is BANG questionnaire with 4 yes/no questions answered by a dedicated health worker that examined the patient: is the body mass index (BMI) higher than $35 \mathrm{~kg} / \mathrm{m}^{2}$, is the patient more than 50 years old, if the measured neck circumference is more than $40 \mathrm{~cm}$, and if the patient's gender is male. High risk for OSA on the STOP-Bang is defined when 3 or more questions are answered positively [20].

\section{Epworth sleepiness scale (ESS)}

The ESS is a self-administered questionnaire for measuring the presence of excessive daytime sleepiness [18]. The patient needs to rate his/her chances of dozing off in 8 common daily situations (reading, watching TV, after meal, etc.) on a scale of $0-3$. If the sum of scores is more than 10 , a patient is considered having excessive daytime sleepiness. The ESS has been previously validated in the Serbian language [33]. 


\section{Anthropometric measurements}

All measurements were performed in patients wearing light clothes, without shoes, before the sleep study. Body weight was measured with digital scale (SE 818, SECA Germany) with $0.1 \mathrm{~kg}$ accuracy, while body height was measured with portable stadiometer (SE 213, SECA Germany) with $0.1 \mathrm{~cm}$ accuracy. Neck, waist and hip circumference were measured using standard non-elastic measuring tape with $0.1 \mathrm{~cm}$ accuracy.

\section{Polysomnography}

All subjects underwent a single-night attended complete polysomnography using a type I polysomnography system (Alice-5 Philips Respironics Inc., Amsterdam, The Netherlands) or single-night attended cardio-respiratory polysomnography using a type 3 polygraphy system (MS-310 Müller \& Sebastiani Elektronik GmbH, Ottobrunn, Germany). Complete polysomnography was performed according to standard techniques, with concurrent monitoring of the electroencephalogram using frontal, central and occipital leads, electro-oculogram, chin electro-myogram, flow (by oronasal thermistor and nasal air pressure transducer), thoracic and abdominal respiratory effort (induction plethysmography), oximetry, body position, and leg electro-myogram. Snoring was recorded by a microphone placed on the anterior neck. Polysomnographic recordings were manually scored in accordance with the American Academy of Sleep Medicine (AASM) manual of scoring sleep and sleep-associated events [34]. Cardiorespiratory polygraphy was performed according to standard techniques, with monitoring of the respiratory flow (by oronasal thermistor), thoracic and abdominal respiratory effort (induction plethysmography belts), oximetry, and body position. Snoring was recorded by an integrated microphone. Type I and type III PSG were both performed in hospital sleep lab settings, with continuous video monitoring. Apnea was defined as a drop in the peak thermal sensor excursion by $90 \%$ or more of baseline, for at least $10 \mathrm{~s}$. Hypopnea was defined as a nasal pressure signal excur- sion drop by $\geq 50 \%$ of baseline, with a $\geq 3 \%$ oxygen desaturation or an arousal [34]. Apnea-hypopnea index (AHI), the number of apneas and hypopneas per hour of sleep, was used for establishing OSA diagnosis and severity. Obstructive sleep apnea was considered mild if AHI was $\geq 5$ and $<15 / \mathrm{h}$, moderate if AHI was $\geq 15$ and $<30 / \mathrm{h}$ and severe if AHI was $\geq 30 / \mathrm{h}$.

\section{Ethical approval}

This study has received ethical approval from the Ethical board of Faculty of Medicine, University of Belgrade, Serbia. The study has been performed in accordance with the ethical standards laid down in the 1964 Declaration of Helsinki and its later amendments.

\section{Statistical analysis}

The validation included evaluation of test-retest reliability, construct validity and discriminant validity. Test-retest reliability was assessed with Cohen's $x$ coefficient. Discriminant validity was evaluated by comparison of healthy and OSA subjects.

Data is presented as mean $(\mathrm{M}) \pm$ standard deviation (SD) for continuous or $\mathrm{N}(\%)$ for categorical data. The T-test and Mann-Whitney U test were used for testing differences between groups. Spearman correlation was used for assessing relationship between scores. The receiver operating characteristic (ROC) curve and Youden index (J) were used for determining best cut off value for different OSA severities. For all scores sensitivity, specificity, positive and negative predictive values and positive and negative likelihood ratios were calculated with $95 \%$ confidence intervals (CI). All data was analyzed in SPSS 20.0 (IBM Corp.) statistical package. All $p$ values less than 0.05 were considered significant.

\section{RESULTS}

\section{Characteristics of study population}

Out of one hundred subjects, 76 patients were overweight $\left(\mathrm{BMI}>25 \mathrm{~kg} / \mathrm{m}^{2}\right)$ and 35 were obese $\left(\mathrm{BMI}>30 \mathrm{~kg} / \mathrm{m}^{2}\right)$. 
Table 1. Demographic and polysomnographic characteristics of the study population - commercial bus and truck male drivers according to STOP-Bang risk group

\begin{tabular}{|c|c|c|c|c|}
\hline \multirow[b]{2}{*}{ Variable } & \multicolumn{3}{|c|}{ Respondents } & \multirow[b]{2}{*}{$\mathrm{p}$} \\
\hline & $\begin{array}{c}\text { total } \\
(\mathrm{N}=100)\end{array}$ & $\begin{array}{c}\text { low risk } \\
(\text { STOP-Bang < 3) } \\
(\mathrm{N}=31)\end{array}$ & $\begin{array}{c}\text { high risk } \\
(\text { STOP-Bang } \geq 3) \\
(\mathrm{N}=69)\end{array}$ & \\
\hline Age [years] $(\mathrm{M} \pm \mathrm{SD})$ & $43.4 \pm 10.7$ & $36.2 \pm 9.5$ & $46.6 \pm 9.5$ & $<0.001$ \\
\hline Body mass index $(\mathrm{BMI})\left[\mathrm{kg} / \mathrm{m}^{2}\right](\mathrm{M} \pm \mathrm{SD})$ & $29.0 \pm 5.7$ & $25.7 \pm 3.9$ & $30.4 \pm 5.8$ & $<0.001$ \\
\hline \multicolumn{5}{|l|}{ Circumference $[\mathrm{cm}](\mathrm{M} \pm \mathrm{SD})$} \\
\hline neck & $40.4 \pm 3.3$ & $38.0 \pm 2.7$ & $41.5 \pm 3.3$ & $<0.001^{\mathrm{a}}$ \\
\hline waist & $102.2 \pm 14.3$ & $94.3 \pm 10.6$ & $106.2 \pm 14.4$ & $<0.001^{\mathrm{a}}$ \\
\hline hip & $104.6 \pm 9.4$ & $100.6 \pm 6.7$ & $106.6 \pm 10.0$ & $0.004^{\mathrm{a}}$ \\
\hline Epworth sleepiness scale (ESS) score $(\mathrm{M} \pm \mathrm{SD})$ & $6.4 \pm 4.4$ & $4.5 \pm 3.3$ & $7.2 \pm 4.6$ & $0.006^{\mathrm{a}}$ \\
\hline \multicolumn{5}{|l|}{ Polysomnography (PSG) parameters $(\mathrm{M} \pm \mathrm{SD})$} \\
\hline Apnea-hypopnea index (AHI) & $12.1 \pm 15.2$ & $3.9 \pm 3.6$ & $15.8 \pm 16.9$ & $<0.001^{\mathrm{b}}$ \\
\hline $\mathrm{AHI}_{\text {supine }}$ & $19.1 \pm 22.5$ & $7.6 \pm 9.2$ & $24.4 \pm 24.8$ & $<0.001^{\mathrm{b}}$ \\
\hline snoring [\%] & $7.5 \pm 10.7$ & $3.2 \pm 4.9$ & $9.5 \pm 12.0$ & $0.001^{\mathrm{b}}$ \\
\hline \multicolumn{5}{|l|}{$\mathrm{SatO}_{2}[\%](\mathrm{M} \pm \mathrm{SD})$} \\
\hline during time in bed & $95.3 \pm 2.0$ & $95.8 \pm 1.6$ & $95.0 \pm 2.1$ & $0.048^{b}$ \\
\hline during sleep (min.) & $85.5 \pm 10.4$ & $88.6 \pm 7.6$ & $84.1 \pm 11.2$ & $0.003^{b}$ \\
\hline
\end{tabular}

STOP-Bang - STOP-Bang questionnaire (snoring, tiredness, observed apnea, blood pressure, body mass index, age, neck circumference, gender). $\mathrm{SatO}_{2}$ - arterial blood oxygen saturation.

$\mathrm{M}$ - mean; SD - standard deviation, min. - minimal value.

a T-test.

${ }^{\mathrm{b}}$ Mann Whitney U test.

Most of the patients (71\%) were married, with children, current or ex-smokers (47\% and 19\%, respectively) that seldom consume alcohol drinks $(75 \%)$. Usual health issues were musculoskeletal pain $(50 \%)$, gastrointestinal disorders (gastritis, reflux oesophagitis) (38\%) and upper respiratory chronic inflammatory disorders (sinusitis, rhinitis, pharyngitis) (37\%). Approximately one-third (34 patients) reported arterial hypertension (HTA), while diabetes mellitus type II was recognized by $8 \%$, and psychiatric problems by $6 \%$ of the participants.

Excessive daytime sleepiness (ESS $\geq 10$ ) was recognized for 21 drivers, mean ESS score was 6.4 (min. 0, max 19). STOP-Bang recognized 69 potential OSA patients. Polysomnography revealed presence of OSA (AHI $\geq 5$ ) in $57 \%$ of the subjects. Eleven patients had a moderate form of OSA and 12 had severe OSA. There was a significant difference in most of the demographic and PSG findings between subjects in high and low risk group according to STOP-Bang. Patients in OSA high risk group were older, with higher BMI, neck, and waist and hip circumference, with more snoring, higher ESS, AHI, and lower mean oxygen saturation values (Table 1).

\section{Reliability}

In order to test reproducibility of the translated questionnaire, all subjects answered the questionnaires on 2 separate occasions 3-9 months apart: during the initial contact and before PSG. Time period between the test and retest 
may seem inadequate, but since there were no interventions in-between, and the questionnaire is a screening tool for a chronic condition (OSA), the level of test-retest reliability was high (Cohen's $x=0.89,95 \%$ CI: 0.79-0.98, $\mathrm{p}<0.001)$. The lower level of agreement was seen on questions about snoring (Cohen's $x=0.80$ ), tiredness (Cohen's $x=0.81$ ), self reported elevated blood pressure (Cohen's $x=0.85$ ) and the objective BMI (Cohen's $x=0.90)$.

\section{Validity}

Epworth sleepiness scale is currently the only formally translated and validated measure used for OSA screening available in the Serbian language.

Mean ESS score for subjects without OSA was 5.95 $(\mathrm{SD}=3.73)$, while for subjects with OSA the average ESS was $6.67(\mathrm{SD}=5.83)$. Eight out of 12 drivers with severe OSA did not report excessive daytime sleepiness. Although we found no significant difference in ESS score between patients with or without OSA ( $p=0.706)$, there was a positive relationship between the ESS score (0-24) and the STOP-Bang score (value 0-8). Spearman's @ coefficient was $\varrho=0.157(\mathrm{p}=0.009)$.

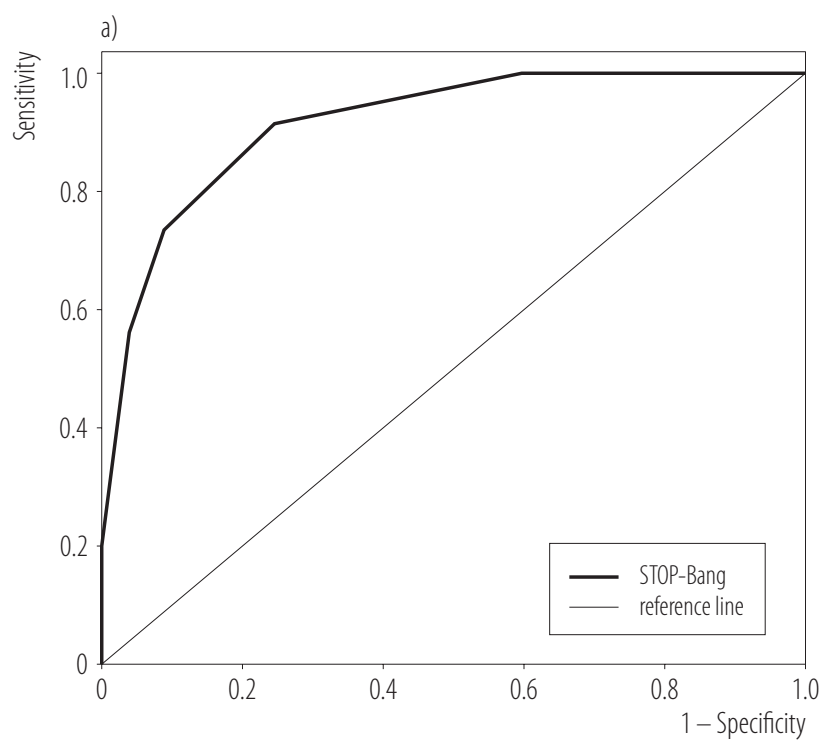

The gold standard for diagnosis of OSA is overnight polysomnography. We correlated the SBQ score to AHI and analyzed if the recommended STOP-Bang score of 3 and more indicated presence of OSA. Use of STOP-Bang questionnaire revealed 69 subjects in high risk for OSA, while the PSG confirmed OSA in 49 of them (71\%). In the low risk group, 23 subjects $(74.2 \%)$ were correctly identified as subjects without OSA (AHI < 5). STOPBang questionnaire $\geq 3$ recognized all patients with severe OSA, but also misclassified 20 patients without OSA as high risk subjects. There was a significant correlation between SBQ score and AHI ( $\varrho=0.667, \mathrm{p}<0.001)$.

The predictive parameters (sensitivity, specificity, positive and negative predictive value, positive and negative likelihood ratio) of SBQ for subjects with different OSA categories are shown in the Table 2.

Best STOP-Bang cut-off value for moderate OSA was between 4 (sensitivity 91\%, specificity $75.3 \%, \mathrm{~J}=0.663$ ) and 5 (sensitivity $74 \%$, specificity $90.9 \%, \mathrm{~J}=0.649$ ). For severe OSA (AHI $\geq 30$ ), 4 out of 8 positive answers yielded the best sensitivity (91.7\%) and specificity (67\%) with Youden index of 0.587 (Figure 1).

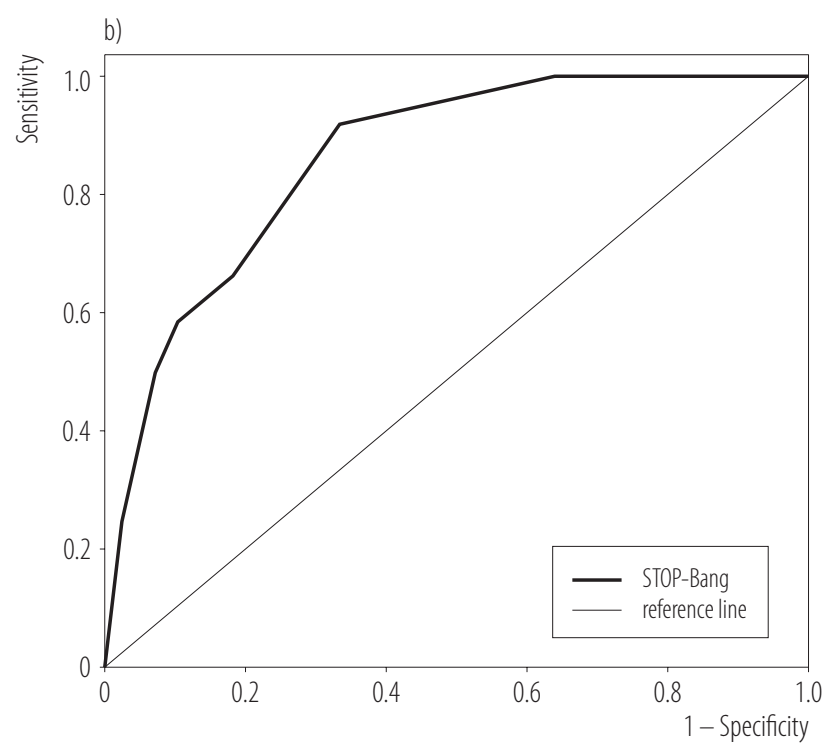

Fig. 1. Receiver operating characteristic (ROC) curves of STOP-Bang questionnaire using a) apnea-hypopnea index (AHI) $\geq 15$ or b) AHI $\geq 30$ cut-off value in study population - commercial bus and truck male drivers 
Table 2. Predictive parameters of STOP-Bang questionnaire $\geq 3$ score for identifying obstructive sleep apnea (OSA) in study population - commercial bus and truck male drivers

\begin{tabular}{|c|c|c|}
\hline Parameter & M & $95 \% \mathrm{CI}$ \\
\hline \multicolumn{3}{|l|}{ Apnea-hypopnea index (AHI) $\geq 5$} \\
\hline sensitivity [\%] & 86.000 & $74.200-93.700$ \\
\hline specificity [\%] & 53.500 & $37.700-68.800$ \\
\hline \multicolumn{3}{|l|}{ likehood ratio } \\
\hline positive & 1.850 & $1.320-2.590$ \\
\hline negative & 0.260 & $0.130-0.530$ \\
\hline \multicolumn{3}{|l|}{ predictive value [\%] } \\
\hline positive (PPV) & 71.000 & $58.800-81.300$ \\
\hline negative (NPV) & 74.200 & $55.400-88.100$ \\
\hline area under the ROC curve (AUC) & 0.804 & $0.720-0.888$ \\
\hline \multicolumn{3}{|l|}{ Apnea-hypopnea index $(\mathrm{AHI}) \geq 15$} \\
\hline sensitivity [\%] & 100.000 & $85.100-100.000$ \\
\hline specificity [\%] & 40.300 & $29.200-52.100$ \\
\hline \multicolumn{3}{|l|}{ likehood ratio } \\
\hline positive & 1.670 & $1.390-2.010$ \\
\hline negative & 0.000 & \\
\hline \multicolumn{3}{|l|}{ predictive value [\%] } \\
\hline positive (PPV) & 33.300 & $22.400-45.700$ \\
\hline negative (NPV) & 100.000 & $88.700-100.000$ \\
\hline area under the ROC curve (AUC) & 0.916 & $0.855-0.976$ \\
\hline \multicolumn{3}{|l|}{ Apnea-hypopnea index $(\mathrm{AHI}) \geq 30$} \\
\hline sensitivity [\%] & 100.000 & $73.300-100.000$ \\
\hline specificity [\%] & 35.200 & $25.300-46.100$ \\
\hline \multicolumn{3}{|l|}{ likehood ratio } \\
\hline positive & 1.540 & $1.320-1.800$ \\
\hline negative & 0.000 & \\
\hline \multicolumn{3}{|l|}{ predictive value [\%] } \\
\hline positive (PPV) & 17.400 & $9.300-28.400$ \\
\hline negative (NPV) & 100.000 & $88.700-100.000$ \\
\hline area under the ROC curve (AUC) & 0.862 & $0.768-0.957$ \\
\hline
\end{tabular}

ROC - receiver operating characteristic.

$\mathrm{M}$ - mean; CI - confidence interval.

\section{DISCUSSION}

The main aim of this study has been to explore psychometric properties of STOP-Bang scoring model (Serbian translation) in a sample of commercial drivers.

Test-retest reliability was adequate (Cohen's $x=0.89$ ), in line with the validation studies for other languages [35]. We used the ESS score for testing construct validity of STOPBang, and found a significant correlation between results. Mean ESS scores for subjects with or without OSA were approximately 6.7 and 6.0, respectively. One-third of the subjects with severe OSA did not report excessive daytime sleepiness. Low ESS was found also in other studies of 
commercial drivers [29,36]. Kales et al. in their review paper [37] presented some of the potential explanations why commercial drivers rarely report excessive sleepiness when screened for OSA.

Considering the STOP-Bang, sensitivity reached $100 \%$ for $\mathrm{AHI} \geq 15$, but the highest specificity was 53.5 for $\mathrm{AHI} \geq 5$. Positive predictive value (PPV) was $71 \%$ for $\mathrm{AHI} \geq 5$, compared to only $17.4 \%$ for $\mathrm{AHI} \geq 30$. In previous studies, sensitivity and specificity of STOP-Bang questionnaire were evaluated in different population samples, ranging from general population, primary health care patients, to surgical patients, patients with chronic conditions and patients referred to sleep clinics. Performance of STOPBang was initially evaluated for surgical patients [20] in comparison to Type I PSG, using 3 AHI cut-offs. With a STOP-Bang score $\geq 3$, sensitivities were $83.6 \%, 92.9 \%$, and $100 \%$, respectively, while the highest specificity $(56.4 \%)$ and PPV (81\%) was at $\mathrm{AHI} \geq 5$.

Silva et al. [21] compared several questionnaires against type III PSG in a large community sample (4770 participants of the Sleep Heart Health Study) and concluded that STOP-Bang had the highest sensitivity in identifying subjects with moderate and severe OSA (87\% and 70.4\%, respectively), with moderate specificity ( $43.3 \%$ and $59.5 \%$, respectively).

In primary health care patients, using the $\mathrm{AHI}>5$ as diagnostic for OSA, STOP-Bang showed sensitivity of $96.4 \%$, with specificity of $24 \%$ and a positive predictive value of $66 \%$ [38]. Validation for patients with chronic and endstage renal disease showed very high sensitivity and low specificity for moderate and severe OSA [23].

STOP-Bang questionnaire was extensively tested for patients referred on suspicion of sleep disorder with different outcomes [35,39-42]. Similar to our results, validation of STOP-Bang in the Portuguese language [35] yielded high sensitivity in all 3 AHI cut-offs, with the highest PPV at AHI $>5$ cut-off. Comparison of several standard questionnaires in sleep clinic settings in Egypt [41], Canada [39] and Greece [42] found that STOP-Bang had very high sensitivity for all AHI cut-offs, with a low specificity. Best sensitivity, specificity and PPV were at AHI $>5$ in Egyptian and Canadian study, and at AHI > 15 in the Greek study. Some researchers experimented with various STOP-Bang cut-off scores. In our study, best sensitivity and specificity were seen at 4 or 5 positive answers for moderate OSA, and 4 answers for severe OSA. In a study performed in army veterans [24], raising the STOP-Bang score from 3 to 5 led to slight decrease in sensitivity, increase in specificity and PPV in screening for moderate OSA. Cowan et al. found that for AHI $>5$ and AHI $>15$ cut-off, the best overall accuracy and PPV was at STOP-Bang of 3 or 6 [40]. Validation of Portuguese STOP-Bang showed that for AHI $>5$ best cut-off was at three, and for higher AHI categories at 4 correct answers [35].

Ozder et al. [30] used ESS and STOP-Bang as screening tools in the population of 618 bus drivers in Turkey, and found high OSA risk in $71.5 \%$, while $48.1 \%$ had excessive sleepiness. There was a positive correlation between sleepiness and OSA risk and sleepiness and reported traffic accidents, but no significant relationship between OSA risk and accidents. In a similar study performed in Lagos, Nigeria [29], STOP-Bang classified $48.8 \%$ of bus drivers as high risk, while ESS recognized $14.4 \%$ as sleepy drivers. Unfortunately, neither of the study protocols included PSG or other objective methods for confirming OSA diagnosis. In an another study performed in a sample of highway bus drivers in Turkey, Firat et al. [28] tested sensitivity and specificity of several questionnaires, including STOP-Bang, by comparing the questionnaire results with daytime polysomnography (performed after night shift). Using the AHI > 15/h cut off, STOP-Bang had an $87 \%$ sensitivity with $47.7 \%$ specificity. Their sample of drivers is very similar to our sample, considering gender, average BMI, neck circumference and even mean AHI. Reported sensitivity and specificity are lower than in our sample of drivers for AHI > 15 cut-off. Also, they reported 
negative predictive value of $76 \%$, while in our study negative predictive value (NPV) reached $100 \%$ for moderate and severe OSA.

In our study, STOP-Bang showed 100\% sensitivity at AHI > 15 using the 3 positive answers cut-off, so this questionnaire may be used as a good screening tool, since the negative result would practically rule out presence of moderate and severe OSA, as required by the EU directive 2014/85/EU [15]. On the other hand, low specificity could lead to sending many false positive subjects to PSG, creating a strong pressure on limited capacities of sleep clinics. Receiver operating characteristic analysis has shown that using the 4 positive answers cut-off could probably provide a better overall accuracy for moderate and severe OSA in our target population.

\section{Limitations of the study}

Our study has several limitations. The study sample consisted of commercial drivers, so there was potential underreporting of subjective sleep problems and their effect on driving due to fear of losing their job. Questionnaire results were compared to a single night PSG, with a possibility that subjects' sleep was worse than usual. It was not feasible to perform a 2-night PSG due to work schedule of drivers. For some patients we performed a type III PSG instead of type I PSG, but it was in attended settings so results were of acceptable quality.

\section{CONSLUSIONS}

STOP-Bang questionnaire (Serbian version) showed good test-retest reproducibility, as well as adequate construct and criterion validity, which supports further use as a screening tool for prediction of obstructive sleep apnea for commercial drivers.

\section{REFERENCES}

1. Epstein LJ, Kristo D, Strollo PJ, Friedman N, Malhotra A, Patil SP, et al. Clinical guideline for the evaluation, mana- gement and long-term care of obstructive sleep apnea in adults. J Clin Sleep Med. 2009;5(3):263-76.

2. Peppard PE, Young T, Barnet JH, Palta M, Hagen EW, Hla KM. Increased prevalence of sleep-disordered breathing in adults. Am J Epidemiol. 2013;177:1006-14, https://doi.org/ 10.1093/aje/kws342.

3. Tregear S, Reston J, Schoelles K, Phillips B. Obstructive sleep apnea and risk of motor vehicle crash: Systematic review and meta-analysis. J Clin Sleep Med. 2009;5(6):573-81.

4. Vennelle M, Engleman HM, Douglas NJ. Sleepiness and sleeprelated accidents in commercial bus drivers. Sleep Breath. 2010;14(1):39-42, https://doi.org/10.1007/s11325-009-0277-z.

5. Karimi M, Eder DN, Eskandari D, Zou D, Hedner JA, Grote L. Impaired vigilance and increased accident rate in public transport operators is associated with sleep disorders. Accid Anal Prev. 2013;51:208-14, https://doi.org/10.1016/j.aap.2012.11.014. 6. Somers VK, White DP, Amin R, Abraham WT, Costa F, Culebras A, et al. Sleep apnea and cardiovascular disease: An American Heart Association/American College of Cardiology Foundation Scientific Statement from the American Heart Association Council for High Blood Pressure Research Professional Education Committee, Council on Clinical Cardiology, Stroke Council, and Council on Cardiovascular Nursing. J Am Coll Cardiol. 2008;52:686-717, https://doi.org/10.1016/j.jacc.2008.05.002.

7. Pamidi S, Aronsohn RS, Tasali E. Obstructive sleep apnea: Role in the risk and severity of diabetes. Best Pract Res Clin Endocrinol Metab. 2010;24(5):703-15, https://doi.org/ 10.1016/j.beem.2010.08.009.

8. Kendzerska T, Gershon A, Hawker G, Tomlinson G, Leung R. Obstructive sleep apnea and incident diabetes. A historical cohort study. Am J Resp Crit Care. 2014;190(2): 218-25, https://doi.org/10.1164/rccm.201312-22090C.

9. Harris RG, Glozier N, Ratnavadivel R, Grunstein RR. Obstructive sleep apnea and depression. Sleep Med Rev. 2009;13:437-44, https://doi.org/10.1016/j.smrv.2009.04.001.

10. Kendzerska T, Gershon A, Hawker G, Leung RS, Tomlinson G. Obstructive sleep apnea and risk of cardiovascular events and all-cause mortality: A decade-long historical 
cohort study. PLoS Med. 2014;11:e1001599, https:/doi. org/10.1371/journal.pmed.1001599.

11. Punjabi NM, Caffo BS, Goodwin JL, Gottlieb DJ, Newman AB, O'Connor GT, et al. Sleep-disordered breathing and mortality: A prospective cohort study. PloS Med. 2009; 6(8):e1000132, https://doi.org/10.1371/journal.pmed.1000132.

12. Gagnon K, Baril A-A, Gagnon J-F, Fortin M, Décary A, Lafond $\mathrm{C}$, et al. Cognitive impairment in obstructive sleep apnea. Pathol Biol. 2014;62(5):233-40, https://doi.org/10. 1016/j.patbio.2014.05.015.

13. Mulgrew A, Nasvadi G, Butt A, Cheema R, Fox N, Fleetham J, et al. Risk and severity of motor vehicle crashes in patients with obstructive sleep apnea/hypopnea. Thorax. 2008; 63(6):536-41, https://doi.org/10.1136/thx.2007.085464.

14. Smolensky MH, di Milia L, Ohayon MM, Philip P. Sleep disorders, medical conditions, and road accident risk. Accid Anal Prev. 2011;43(2):533-48, https://doi.org/10.1016/ j.aap.2009.12.004.

15. Commission Directive 2014/85/EU of 1 July 2014 amending Directive 2006/126/EC of the European Parliament and of the Council on driving licences. Off J Eur Union L 194, p. 10-13 (Jul 2, 2014).

16. Ramachandran SK, Josephs LA. A meta-analysis of clinical screening tests for obstructive sleep apnea. Anesthesiology. 2009;110:928-39, https://doi.org/10.1097/ALN.0b013 e31819c47b6.

17. Balk EM, Moorthy D, Obadan NO, Patel K, Ip S, Chung M, et al. Diagnosis and Treatment of Obstructive Sleep Apnea in Adults. Comparative Effectiveness Review No. 32. (Prepared by Tufts Evidence-based Practice Center under Contract No. 290-2007-10055-1). AHRQ Publication No. 11-EHC052-EF [Internet]. Rockville: Agency for Healthcare Research and Quality; 2011 [cited 2016 Jan 7]. Available from: www.effectivehealthcare.ahrq.gov/reports/ final.cfm.

18. Johns M. A new method for measuring daytime sleepiness: The Epworth Sleepiness Scale. Sleep. 1991;14(6):540-5, https://doi.org/10.1093/sleep/14.6.540.
19. Roure N, Gomez S, Mediano O, Duran J, Peña MDL, Capote F, et al. Daytime sleepiness and polysomnography in obstructive sleep apnea patients. Sleep Med. 2008;9(7): 727-31, https://doi.org/10.1016/j.sleep.2008.02.006.

20. Chung F, Yegneswaran B, Liao P, Chung SA, Vairavanathan S, Islam S, et al. STOP questionnaire: A tool to screen patients for obstructive sleep apnea. Anesthesiology. 2008;108: 812-21, https://doi.org/10.1097/ALN.0b013e31816d83e4.

21. Silva GE, Vana KD, Goodwin JL, Sherrill DL, Quan SF. Identification of patients with sleep disordered breathing: Comparing the Four-Variable screening tool, STOP, STOPBang, and Epworth Sleepiness Scales. J Clin Sleep Med. 2011;7(5):467-72, https://doi.org/10.5664/jcsm.1308.

22. Dias RA, Hardin KA, Rose H, Agius MA, Apperson ML, Brass SD. Sleepiness, fatigue, and risk of obstructive sleep apnea using the STOP-Bang questionnaire in multiple sclerosis: A pilot study. Sleep Breath. 2012;16:1255-65, https:// doi.org/10.1007/s11325-011-0642-6.

23. Nicholl DDM, Ahmed SB, Loewen AHS, Hemmelgarn BR, Sola DY, Beecroft JM, et al. Diagnostic value of screening instruments for identifying obstructive sleep apnea in kidney failure. J Clin Sleep Med. 2013;9(1):31-8, https://doi. org/10.5664/jcsm.2334.

24. Kunisaki KM, Brown KE, Fabbrini AE, Wetherbee EE, Rector TS. STOP-Bang questionnaire performance in a veterans affairs unattended sleep study program. Ann Am Thorac Soc. 2014;11(2):192-7, https://doi.org/10.1513/Annals ATS.201305-134OC.

25. Boynton G, Vahabzadeh A, Hammoud S, Ruzicka DL, Chervin RD. Validation of the STOP-Bang questionnaire among patients referred for suspected obstructive sleep apnea. J Sleep Disord Treat Care. 2013;2:4, https://doi. org/10.4172/2325-9639.1000121.

26. Talmage JB, Hudson TB, Hegmann KT, Thiese MS. Consensus criteria for screening commercial drivers for obstructive sleep apnea: Evidence of efficacy. J Occup Environ Med. 2008;50:324-9, https://doi.org/10.1097/JOM.0b013e31 $81617 \mathrm{ab} 8$. 
27. Parks P, Durand G, Tsismenakis AJ, Vela-Bueno A, Kales S. Screening for obstructive sleep apnea during commercial driver medical examinations. J Occup Environ Med. 2009;51: 275-82, https://doi.org/10.1097/JOM.0b013e31819eaaa4.

28. Firat H, Yuceege M, Demir A, Ardic S. Comparison of four established questionnaires to identify highway bus drivers at risk for obstructive sleep apnea in Turkey. Sleep Biol Rhythms. 2012;10(3):231-6, https://doi.org/10.1111/j.14798425.2012.00566.x.

29. Ozoh OB, Okubadejo NU, Akanbi MO, Dania MG. Highrisk of obstructive sleep apnea and excessive daytime sleepiness among commercial intra-city drivers in Lagos metropolis. Niger Med J. 2013;54(4):224-9, https://doi.org/ 10.4103/0300-1652.119607.

30. Ozder A, Gunay E, Huseyin Eker H, Saris Ulasli S. Excessive daytime sleepiness among Turkish public transportation drivers: A risk for road traffic accidents? Acta Med Mediterr. 2014;30:1121.

31. Burns N. An integrative review of screening for obstructive sleep apnea in commercial vehicle drivers. Workplace Health Saf. 2014;62(3):114-20, https://doi.org/10.1177/216 507991406200305 .

32. Beaton DE, Bombardier C, Guillemin F, Ferraz MB. Guidelines for the process of cross-cultural adaptation of selfreport measures. Spine. 2001;25(24):3186-91, https://doi. org/10.1097/00007632-200012150-00014.

33. Kopitovic I, Trajanovic N, Prodic S, Drvenica MJ, Ilic M, Kuruc V, et al. The Serbian version of the Epworth Sleepiness Scale. Sleep Breath. 2011;15(4):775-80, https://doi. org/10.1007/s11325-010-0435-3.

34. Iber C, Ancoli-Israel S, Chesson A, Quan SF. The AASM manual for the scoring of sleep and associated events: Rules, terminology and technical specifications. Westchester: American Academy of Sleep Medicine; 2007.
35. Reis R, Teixeira F, Martins V, Sousa L, Batata L, Santos C, et al. Validation of a Portuguese version of the STOP-Bang questionnaire as a screening tool for obstructive sleep apnea: Analysis in a sleep clinic. Rev Port Pneumol. 2015;21(2): 61-8, https://doi.org/10.1016/j.rppnen.2014.04.009.

36. Sharwood LN, Elkington J, Stevenson M, Grunstein RR, Meuleners L, Ivers RQ, et al. Assessing sleepiness and sleep disorders in Australian long-distance commercial vehicle drivers: Self-report versus an "at home" monitoring device. Sleep. 2012;35:469-75, https://doi.org/10.5665/sleep.1726.

37. Kales SN, Straubel M. Obstructive sleep apnea in North American commercial drivers. Ind Health. 2013;52(1): 13-24, https://doi.org/10.2486/indhealth.2013-0206.

38. Cruces-Artero C, Martin-Miguel MV, Herves-Beloso C, Lago-Deibe F, Hernaiz-Valero S, Claveria-Fontan A, et al. Validation of the STOP and STOP BANG Questionnaire in Primary Health Care. J Sleep Res. 2012;21:226.

39. Pereira EJ, Driver HS, Stewart SC, Fitzpatrick MF. Comparing a combination of validated questionnaires and level III portable monitor with polysomnography to diagnose and exclude sleep apnea. J Clin Sleep Med. 2013;9(12):1259-66, https://doi.org/10.5664/jcsm.3264.

40. Cowan DC, Allardice G, MacFarlane D, Ramsay D, Ambler H, Banham S, et al. Predicting sleep disordered breathing in outpatients with suspected OSA. BMJ Open. 2014;4: e004519, https://doi.org/10.1136/bmjopen-2013-004519.

41. El-Sayed IH. Comparison of four sleep questionnaires for screening obstructive sleep apnea. Egypt J Chest Dis Tuberc. 2012;61(4):433-41, https://doi.org/10.1016/j.ejcdt.2012.07.003.

42. Pataka A, Daskalopoulou E, Kalamaras G, Fekete Passa K, Argyropoulou P. Evaluation of five different questionnaires for assessing sleep apnea syndrome in a sleep clinic. Sleep Med. 2014;15(7):776-81, https://doi.org/10.1016/ j.sleep.2014.03.012.

This work is available in Open Access model and licensed under a Creative Commons Attribution-NonCommercial 3.0 Poland License - http://creativecommons.org/ licenses/by-nc/3.0/pl/deed.en. 